\title{
Physiological effects of selective tibial neurotomy on lower limb spasticity
}

\author{
A Fève, P Decq, P Filipetti, J Verroust, A Harf, J P N'Guyen, Y Keravel
}

\begin{abstract}
Objectives-To assess by electrophysiology the effect of tibial selective neurotomy on muscle imbalance of the spastic ankle. Method-The amplitudes of the $H$ reflexes, $M$ responses (muscle contractions recorded after stimulation of the tibial nerve), and Hmax:Mmax ratio were recorded in 12 patients with chronic lower limb spasticity, before and one month after tibial selective neurotomy. Recordings were done on medial and lateral gastrocnemius and soleus muscles.

Clinical evaluation was done with both global (Held's score) and analytical tests (step measurements, gait velocity, and ankle angulation during active and passive movements).

Results-After neurotomy, gait improved in all patients. Held 's score of spasticity was better in all patients. Active dorsiflexion of the ankle was unchanged in three patients, but the others improved by $5^{\circ}$ to 12 ${ }^{\circ}$. Hmax, Mmax, and Hmax:Mmax ratios were lower. The Hmax on the gastrocnemius muscle, clinical strength, Mmax of all the muscles, and Hmax:Mmax ratio for the soleus and lateral gastrocnemius muscle were significantly lower after surgery.

Conclusion-There was an improvement of clinical and electrophysiological spastic indices after selective tibial neurotomy. Neurotomy acted not only on motor neurons by decreasing strength, but also the reflex enlargement by decreasing sensory afferents.
\end{abstract}

Service de

Neurochirurgie et d'Explorations

Fonctionnelles, Hopital

Henri Mondor, Créteil,

France

A Fève

P Decq

P Filipetti

J Verroust

A Harf

J P N'Guyen

Y Keravel

Correspondence to: Dr A Fève, Service de Neurochirurgie, Hopital Henri Mondor, Avenue de Lattre de Tassigny. 94010 Créteil. France.

Received 28 August 1997 and in revised form 16 April 1997

Accepted 21 April 1997
(F Neurol Neurosurg Psychiatry 1997;63:575-578)

Keywords: neurotomy; spasticity; $\mathrm{H}$ reflex; reflexology

Spasticity of the lower limbs leads to an abnormal tonic balance between agonist and antagonist muscles with cocontraction of both agonist and antagonist muscles during gait. ${ }^{1}$ Peripheral surgical techniques are designed to restore the balance between agonists and antagonist muscles by decreasing the tone of the spastic muscles. Selective tibial neurotomy consists of section of part of the motor fascicles of the nerve. The aim of neurotomy is to reduce spasticity in this muscle, without excessive impairment of motor strength. It has been shown to be an effective technique to treat varus equinus posture of the spastic foot. ${ }^{23}$
This study was conducted to assess the changes of muscular strength by clinical examination and the neurophysiological indices of spasticity in varus equinus posture of spastic foot after tibial neurotomy. To determine whether selective tibial neurotomy could act on spinal circuitry, Hmax and Hmax:Mmax ratios were recorded after stimulation of the afferent motor neuron. The $\mathrm{H}$ reflex was recorded by measuring the muscle contraction after stimulation of the tibial nerve in the fossa poplitea. This stimulation also allowed the recording of the $M$ response on the muscle at a greater amplitude of stimulation. As lower stimulation intensities elicit the monosynaptic reflex, measurement of the $\mathrm{H}$ reflex evaluates the activity of the monosynaptic myotatic reflex, ${ }^{4}$ and Hmax:Mmax can be considered to be a measure of the excitability of the motor neuron pool. ${ }^{5}$

\section{Patients and methods}

PATIENTS

Twelve patients (eight male, four female, mean age 35.8 (SD 15.3) years) were studied with their informed consent, before and after selective tibial neurotomy. Patients had severe chronic spasticity of one lower limb for a mean duration of 52.7 (SD 22.1) months. Table 1 shows the aetiology of this spasticity and patients' clinical characteristics. Patients were examined one week before and one month after surgery.

\section{CLINICAL EVALUATION}

All the patients complained of gait difficulties. Passive and active ranges of movement were determined and muscle testing (MRC strength scale) of the flexor muscles of the ankle was performed. Held's score ${ }^{6}$ and the Ashworth score $^{7}$ were determined. Held's score evaluates the stretch reflex and the Ashworth score evaluates the muscle tone. In Held's score, the stretch reflex is evaluated and scaled from 0 to $5(0=$ no muscular contraction, $1=$ visible muscle contraction, $3=$ contraction lasting a few seconds, $4=$ permanent clonus, $5=$ rigidity without passive mobilisation. These measures were compared with the global ability to walk and the need for a splint.

To transiently abolish spasticity by selective blockade of one nerve trunk, an anaesthetic block of the tibialis nerve was performed. If positive, this confirmed the usefulness of denervation. The anaesthetic block was performed locally by percutaneously inserting a sheathed needle. Electrical stimulation by this needle technique allowed the injection of $5 \mathrm{ml}$ 
lidocaine $(1.5 \%)$ as close as possible to the tibialis nerve trunk in the fossa poplitea. If the results of the blockade were in agreement with the expected effects of surgery (disappearance of the equine posture and lack of retraction of the Achilles tendon), neurotomy was scheduled.

Velocity, rhythm, and gait cycle length were evaluated on video before and after neurotomies

SURGICAL PROCEDURE

The surgical procedure consisted of partial selective section of the nerves supplying the soleus and medial and lateral heads of the gastrocnemius muscles. Patients were operated on in a prone position, with the knee slightly flexed at $10^{\circ}$. The leg was draped to allow a sufficient view of the foot during stimulation. The skin incision was performed vertically from the transverse popliteal line, to allow identification of all tibial nerve branches. The branches to the soleus and medial and lateral gastrocnemius muscles were exposed and dissected, and partially sectioned using an operating microscope. Tripolar stimulation was used to test the residual strength of the denervated muscle, and to stop the section at the desired level of muscle strength. The drugs used during surgery were halothane, nitrous oxide, and fentanyl. No curarisation was added after induction of anaesthesia.

ELECTROPHYSIOLOGICAL TESTS BEFORE AND AFTER SURGERY

The $M$ responses and $\mathrm{H}$ reflexes were recorded one week before and one month after surgery by the same examiner. Before surgery, $H$ reflexes, $M$ responses, and the Hmax:Mmax ratio were measured for both the spastic and the normal leg. The $\mathrm{H}$ reflexes in the soleus were elicited in a manner similar to that described by Hugon et al. ${ }^{8}$ Stimulation was delivered to the tibial nerve over the popliteal fossa with the proximal cathode and the active surface electrodes were located over the soleus muscle and gastrocnemii with reference electrodes placed distally on the muscles. The active recording electrode was placed on the medial or the lateral head of gastrocnemius muscles.

Duration of stimulus was $1 \mathrm{~ms}$ with a frequency of $0.6 \mathrm{~Hz}$. The band pass ranged from $8 \mathrm{~Hz}$ to $8 \mathrm{kHz}$. The $\mathrm{H}$ reflex was determined usually at the lower intensities of

Table 1 Clinical characteristics of the patients

\begin{tabular}{llllll}
\hline Cases & Sex & Age $(y)$ & Aetiology & $\begin{array}{l}\text { Duration of follow } \\
\text { up (months) }\end{array}$ & $\begin{array}{l}\text { Duration of the } \\
\text { disease (months) }\end{array}$ \\
\hline 1 & F & 63 & Stroke & 7 & 96 \\
2 & M & 34 & HI & 8 & 18 \\
3 & M & 34 & HI & 2 & 39 \\
4 & M & 23 & Stroke & 12 & 48 \\
5 & M & 39 & HI & 5 & 60 \\
6 & M & 19 & HI & 3 & 49 \\
7 & M & 55 & SI & 1 & 24 \\
8 & M & 70 & Stroke & 1 & 72 \\
9 & F & 44 & Stroke & 6 & 72 \\
10 & F & 6 & Stroke & 6 & 72 \\
11 & F & 25 & Stroke & 3 & 19 \\
12 & M & 18 & HI & 1 & \\
\hline
\end{tabular}

$\mathrm{HI}=$ head injury; $\mathrm{SI}=$ spinal injury. stimulation and the stimulation was increased to obtain an Mmax response on the same muscles.

STATISTICAL ANALYSIS

The $M$ response, $H$ reflexes, and Hmax;Mmax ratio were compared before surgery between the affected side and the normal or less affected limb by Student's $t$ test, and on the affected limb before and after surgery by a paired Student's $t$ test.

FOLLOW UP

The patients were re-examined one month after surgery. The same tests and clinical evaluation were performed. The mean duration of follow up is currently 4.9 (SD 2) months after surgery.

\section{Results}

No local or general complications occurred during or after surgery over the follow up period.

CLINICAL CHARACTERISTICS BEFORE AND AFTER SURGERY (TABLE 2)

Analytical indices of spasticity were improved. Before surgery, Held's score was 2 for patient 8 , 3 for patients 2 and 9 , and 4 for the other nine patients. After surgery, Held's score was evaluated as 0 - that is, there was no stretch of the ankle after surgery - in all but one of the patients (patient 3), who had a Held's score of 2 . There were no significant changes in velocity (mean velocity before surgery $1.65 \mathrm{~km} / \mathrm{h}$ and after $1.62 \mathrm{~km} / \mathrm{h}$ ), rhythm (mean walking rhythm before surgery 37.21 steps/min and after 35.38 steps $/ \mathrm{mn}$ ), and gait cycle length (mean length before surgery $70.33 \mathrm{~cm}$ and after $72.02 \mathrm{~cm}$ ).

PASSIVE ANKLE ANGULATION

No patient had excessive limitation of the passive motility of the ankle before surgery, allowing the foot to be plantar grade in stance. Patients 1, 3, 4, 6, 8, and 9 had a dorsiflexion at $0^{\circ}$, patients $2,5,7,10$, and 11 at $+10^{\circ}$, and patient 12 at $+12^{\circ}$.

Passive mobilisation remained unchanged after surgery.

\section{ACTIVE ANKLE MOBILISATION}

Active dorsiflexion was significantly improved after surgery.

Before surgery, three patients $(1,4$, and 5) had no voluntary active dorsiflexion. For these patients, antagonist muscle contractions were only obtained during synkinetic movements. Six patients $(3,6,7,8,10$, and 12$)$ had a limitation of $-10^{\circ}$ of dorsiflexion, and two had $0^{\circ}$ of dorsiflexion (patients 2 and 9). Finally, patient 11 had a dorsiflexion of $10^{\circ}$ before surgery.

After surgery, patients 1,4 , and 5 had no change and patient 11 had impaired dorsiflexion (dorsiflexion evaluated at $5^{\circ}$ ). By contrast, an improvement was found in the other eight patients. Patients $3,8,10$, and 12 had a dorsiflexion of $0^{\circ}$, patient 2 of $+5^{\circ}$, and patients 6,7 , and 9 of $+10^{\circ}$. 
Table 2 Clinical evaluation of spasticity before and after surgery

\begin{tabular}{|c|c|c|c|c|c|c|c|c|c|}
\hline Patients & $\begin{array}{l}\text { Passive ankle } \\
\text { mobility before } \\
\text { surgery } \\
\text { (degrees) }\end{array}$ & $\begin{array}{l}\text { Spasticity } \\
\text { before surgery } \\
\text { (Held) }\end{array}$ & $\begin{array}{l}\text { Spasticity after } \\
\text { surgery (Held) }\end{array}$ & $\begin{array}{l}\text { Active ankle } \\
\text { mobility before } \\
\text { surgery ( ) }\end{array}$ & $\begin{array}{l}\text { Active ankle } \\
\text { mobility after } \\
\text { surgery }\left({ }^{\circ}\right)\end{array}$ & $\begin{array}{l}\text { Presence of } \\
\text { splint before } \\
\text { surgery }\end{array}$ & $\begin{array}{l}\text { Presence of } \\
\text { splint after } \\
\text { surgery }\end{array}$ & $\begin{array}{l}\text { Triceps strength } \\
\text { before surgery }\end{array}$ & $\begin{array}{l}\text { Triceps strength } \\
\text { after surgery }\end{array}$ \\
\hline 1 & 0 & 4 & 0 & 0 & 0 & 0 & 0 & 5 & 2 \\
\hline 2 & 10 & 3 & 0 & 0 & 5 & 1 & 0 & 5 & 3 \\
\hline 3 & 0 & 4 & 2 & -10 & 0 & 0 & 0 & 5 & 3 \\
\hline 4 & 0 & 4 & 0 & 0 & 0 & 1 & 1 & - & - \\
\hline 5 & 10 & 4 & 0 & 0 & 0 & 1 & 1 & 4 & 2 \\
\hline 6 & 0 & 4 & 0 & -10 & 10 & 0 & 0 & 5 & 3 \\
\hline 7 & 10 & 4 & 0 & -10 & 10 & 0 & 0 & 5 & 2 \\
\hline 8 & 0 & 2 & 0 & -10 & 0 & 1 & 0 & 4 & 2 \\
\hline 9 & 0 & 3 & 0 & 0 & 10 & 1 & 0 & 4 & 2 \\
\hline 10 & 10 & 2 & 0 & -10 & 0 & 1 & 0 & 5 & 3 \\
\hline 11 & 10 & 4 & 0 & 10 & 5 & 1 & 0 & 4 & 2 \\
\hline 12 & 20 & 4 & 0 & -10 & 0 & 1 & 0 & 5 & 2 \\
\hline
\end{tabular}

MUSCULAR STRENGTH

Before surgery, one patient (4) had an untestable triceps. The strength of the triceps surae was normal in patients $1,2,3,6,7,10$, and 12 and subnormal in patients $5,8,9$, and 11. However, testing disclosed a loss of strength after surgery with a score of 2 or 3 one month after surgery. For patient 4 , no contractions were found in the triceps surae before or after surgery.

CLINICAL EFFECTS

All 12 patients experienced the greatest mobility of the ankle during walking after surgery. This was shown by active and passive angulations of the ankle before and after surgery. Before surgery, eight patients needed a splint to compensate for their equinus posture of the ankle. After surgery, only two patients still required a splint. These patients also had no voluntary active dorsiflexion before surgery (patients 4 and 5).

\section{ELECTROPHYSIOLOGICAL INDICES BEFORE}

SURGERY

In all patients, the Hmax:Mmax ratio was significantly $(t$ test, $\mathrm{P}<0.05)$ greater on the affected side than on the less spastic or normal side for the medial head of the gastrocnemius and soleus. For the lateral head of the gastrocnemius, Hmax:Mmax was lower on the normal side, but this difference was not significant.

The Mmax response of the soleus was not significantly different between the normal leg and the spastic leg for all patients, although it was lower on the pathological side. The Mmax on the gastrocnemius was not significantly different between the normal and the spastic side.

The Hmax of the medial head of the gastrocnemius was significantly greater on the affected side, although no significant differences were found between the affected and non-affected soleus and the lateral head of the gastrocnemius.
COMPARISON BETWEEN ELECTROPHYSIOLOGICAL TESTS BEFORE AND AFTER SURGERY

Table 3 shows the results.

The $\mathrm{H}$ reflexes were lower after surgery (0.24 (SEM 0.19)) than before (4.30 (SEM $1.70)$ ) in the soleus $(\mathrm{P}<0.001)$, medial head of the gastrocnemius (0.23 (SEM 0.12) v 2.60 (SEM 1.50); $\mathrm{P}<0.01$ ), and lateral head of the gastrocnemius (0.68 (SEM 0.83) v 2.7 (SEM 1.2) with significant differences $(\mathrm{P}<0.01)$.

The $M$ response decreased after surgery for all muscles, but by various amounts. The greatest change before and after surgery was found in the soleus, from 7.2 (SEM 2.0) $\mathrm{mV}$ to 1.12 (SEM 0.59) $(\mathrm{P}<0.0001)$, but a pronounced change was also noted in the lateral head of the gastrocnemius, from 5.4 (SEM 1.4) to 2.42 (SEM 2.1) $\mathrm{mV}(\mathrm{P}<0.05)$. The decrease of Mmax in the medial head of the gastrocnemius was also significant $(P<0.01)$.

The Hmax:Mmax ratio decreased significantly for the soleus $(P<0.01)$ and the lateral head of the gastrocnemius $(\mathrm{P}<0.05)$, but not for the medial gastrocnemius. In three patients (3, 4, and 5), the Hmax:Mmax ratio was greater after surgery and in one patient (6), it remained the same. However, these four patients obtained a good postsurgical clinical result.

\section{Discussion}

Our study confirms the effectiveness of tibial neurotomy in the treatment of gait disorders related to spastic equinus varus posture of the foot. All patients obtained a real gait improvement, seven for walking and three for clonus of the foot. The benefit was always associated with various degrees of foot plantar flexion, suggesting that the positive effect of neurotomy could be linked to the presence of weakness of the extensors. The neurotomy thus restores the balance between agonists and antagonist muscles.

Table 3 Comparison of electrophysiological variables before and after surgery

\begin{tabular}{|c|c|c|c|c|c|c|c|c|}
\hline$H-S$ & $M-S$ & $H: M-S$ & $H-M G$ & $M-M G$ & $H: M-M G$ & $H-L G$ & $M-L G$ & $H: M-L G$ \\
\hline $4.3 \quad(1.7)$ & $7.2 \quad(2.0)$ & $0.61(0.225)$ & $2.6 \quad(1.5)$ & $\begin{array}{l}\text { Before surgery } \\
7.8 \quad(3.0) \\
\text { After surgery }\end{array}$ & $0.37(0.22)$ & $2.7 \quad(1.2)$ & $5.43(1.63)$ & $0.51(0.26)$ \\
\hline $0.24(0.19)$ & $1.12(0.59)$ & $0.55(0.19)$ & $0.23(0.123)$ & $\begin{array}{c}1.65(1.61) \\
\text { Pvalue }\end{array}$ & $0.43(0.34)$ & $0.68(0.83)$ & $2.42(2.115)$ & $0.22(0.17)$ \\
\hline$<0.001$ & $<0.0001$ & $<0.01$ & $<0.01$ & $<0.001$ & NS & $<0.01$ & $<0.05$ & $<0.05$ \\
\hline
\end{tabular}

Values are means (SEM).

$\mathrm{H}=\mathrm{H}$ max; $\mathrm{M}=\mathrm{Mmax} ; \mathrm{H}: M=\mathrm{Hmax}: M \max ; \mathrm{S}=$ soleus; $M G=$ medial head of gastrocnemius; $\mathrm{LG}=$ lateral head of gastrocnemius. 
Motor strength before surgery was difficult to assess because of concomitant spasticity. Weakness after surgery did not worsen the gait because of the specificity of the triceps surae action during walking. In normal gait, the triceps surae contract eccentrically during the second wheel of the swing phase, to act as a breaking mechanism. In our patients, gait was so slow initially and after surgery, that this breaking was not necessary. ${ }^{9}$

All spastic patients in our study had a greater Mmax:Hmax ratio for the spastic limb, confirming the value of this measurement in spasticity testing before and after treatment. ${ }^{5}$ This is in agreement with several studies and suggests that Hmax:Mmax is a good indicator of spasticity, and especially of the excitability of monosynaptic myotatic reflexes. ${ }^{10}$ Changes in electrophysiological indices were seen to parallel the clinical improvement. The Hmax and Mmax decreased significantly after surgery, suggesting that neurotomy did not act exclusively by decreasing strength, but also by interruption of the monosynaptic reflex pathway. Neurotomy partly interrupts both afferent (I $\alpha$ fibres) and efferent (axons of motor neurons) fibres of the myotatic reflex, and thus lowers the spastic component rather than motor strength. This was confirmed by the decrease of Hmax:Mmax in the soleus and lateral gastrocnemius. The lack of variation of Hmax:Mmax on the medial gastrocnemius, despite the clinical improvement and the decrease of Mmax and Hmax, suggested that this muscle head is less involved in spasticity, but essentially contributes to strength. In this study, the follow up was brief and must be re-evaluated to confirm the improvement. It suggests that in varus equinus spastic foot electrophysiological markers of spasticity such as $\mathrm{H}$ reflex and $M$ response, and Hmax:Mmax, were improved after selective tibial neurotomy. The clinical benefit was correlated with electrophysiological measurements of spasticity. Further studies, based on electrophysiological monitoring, should test the hypothesis of a more selective section which avoids a loss of muscle strength.

1 Knutsson E, Richards C. Different types of disturbed motor control in gait of hemiparetic patients. Brain 1979;102:405-30.

2 Stoffel A. The treatment of spastic contractures. Am $\mathcal{J}$ Orthop Surg 1912;10:611-44.

3 Sindou M, Mertens P. Selective neurotomy of the tibial Sindou $M$, Mertens P. Selective neurotomy of the tibial
nerve for the treatment of the spastic foot. Neurosurgery nerve for the treatm
1988;23:738-44.

4 Hoffman P. Demonstration eines Hemmungsreflexes in menschlichen Rückenmark. Zeitschrift fur Biologie 1920;70: 515-24.

5 Angel RW, Hoffmann WW. The $\mathrm{H}$ reflex in normal spastic and rigid subjects. Arch Neurol 1963;8:591-6.

6 Sindou M, Millet MF. Quantification of spasticity and limb function (based on clinical examination and directed to adult patients). In: Sindou M, Abbott R, Kéravel Y, eds. Neurosurgery for spasticity. Wien: Springer-Verlag, 1991:4751 .

7 Ashworth B. Preliminary trial of carisoprodol in multiple sclerosis. Practitioner 1964;192:540-2.

8 Hugon M. Methodology of the Hoffmann reflex in man. In: Desmedt JE, ed. New developments in electromyography and Desmedt JE, ed. New developments in electromyography and
clinical neurophysiology. Vol 3. Basel: Karger, 1973, 277-93.

9 Perry J. Gait analysis: normal and pathological function. Phorofare, NJ: Slack, 1992.

10 Garcia Mullin R, Mayer RF. H reflexes in acute and chronic hemiplegia. Brain 1972;95:559-72. 